\title{
A Mini-Review and Perspective on Current Best Practice and Emerging Industry 4.0 Methods for Risk Reduction in Advanced Composites Manufacturing
}

\author{
Bryn Crawford ${ }^{1}$, Hamid Khayyam², Abbas S. Milani ${ }^{*}$ \\ ${ }^{1}$ Composites Research Network, School of Engineering, University of British Columbia, Kelowna, Canada \\ ${ }^{2}$ School of Engineering, RMIT University, Melbourne, Melbourne, Australia \\ Email: ^abbas.milani@ubc.ca
}

How to cite this paper: Crawford, B., Khayyam, H. and Milani, A.S. (2021) A Mini-Review and Perspective on Current Best Practice and Emerging Industry 4.0 Methods for Risk Reduction in Advanced Composites Manufacturing. Open Journal of Composite Materials, 11, 31-45. https://doi.org/10.4236/ojcm.2021.112004

Received: February 27, 2021

Accepted: April 17, 2021

Published: April 20, 2021

Copyright $\odot 2021$ by author(s) and Scientific Research Publishing Inc. This work is licensed under the Creative Commons Attribution International License (CC BY 4.0).

http://creativecommons.org/licenses/by/4.0/

(c) (i) Open Access

\begin{abstract}
The manufacturing of composite structures is a highly complex task with inevitable risks, particularly associated with aleatoric and epistemic uncertainty of both the materials and processes, as well as the need for in-situ decision-making to mitigate defects during manufacturing. In the context of aerospace composites production in particular, there is a heightened impetus to address and reduce this risk. Current qualification and substantiation frameworks within the aerospace industry define tractable methods for risk reduction. In parallel, Industry 4.0 is an emerging set of technologies and tools that can enable better decision-making towards risk reduction, supported by data-driven models. It offers new paradigms for manufacturers, by virtue of enabling in-situ decisions for optimizing the process as a dynamic system. However, the static nature of current (pre-Industry 4.0) best-practice frameworks may be viewed as at odds with this emerging novel approach. In addition, many of the predictive tools leveraged in an Industry 4.0 system are black-box in nature, which presents other concerns of tractability, interpretability and ultimately risk. This article presents a perspective on the current state-of-the-art in the aerospace composites industry focusing on risk reduction in the autoclave processing, as an example system, while reviewing current trends and needs towards a Composites 4.0 future.
\end{abstract}

\section{Keywords}

Advanced Composites Manufacturing, Quality Control, Uncertainty, Knowledge Engineering, Industry 4.0, Machine Learning, Limited Data Modeling 


\section{Introduction}

Composite materials are already commonplace in many modern industries, including aerospace, automotive, sports goods, construction and many more [1]. This is in large part due to their high structural performance (e.g. stiffness-to-weight and strength-to-weight ratios), lower manufacturing costs by virtue of co-developing the structure's shape and properties in the same process, and lower maintenance costs [2]. This is further exemplified with products that are large with complex geometries [1] [3], such as aircraft control surfaces or wing structures.

However, despite the many benefits and continued successes of composites, there are significant challenges that manufacturers must still overcome to realize commercially viable and defect-free structures. Part of this is due to the high degree of aleatoric and epistemic uncertainty present within the materials and process [4] [5], which limits the ability to fully model and hence control the process outcomes of significance ahead of time. Further, the multi-physics nature of composites manufacturing, combined with the undelineated approach of forming the shape and material properties of a part at the same time during production [6], adds complexity to the task. To address these challenges and associated risks, a combination of tools and heuristic-based policies within each factory can provide a framework to better understand these systems and embed confidence in the decision-making process. Tools may include process models (e.g. Convergent Manufacturing Technology $\left.{ }^{(}\right)$COMPRO [7], ESI ${ }^{\complement}$ PAM-COMPOSITES [8]), combined with experimental approaches to form frameworks such as structural substantiation and process certification [9]. However, these approaches are typically offline in nature and are not sufficient to address contexts in which rapid decision-making is needed, such as the "bus stop" autoclave cure cycles employed in the aerospace industry.

Figure 1 provides a visual example of this activity, in which parts with different configurations are cured in the same pressure vessel, where different thermal and physical responses to the same cure cycle environment are also expected. Modern technological advancements have introduced capabilities that allow for in-situ data collection, modeling and decision-making at scale, in order to improve factory objectives, such as reducing manufacturing failure rates [10] [11]. The aggregation of such data has been forecast to enable significant improvements in net productivity [12] [13], through initiatives such as Industry 4.0, which constructs closed-loop systems of data collection, screening, model training, testing and deployment for in-situ decision-making [11].

Industry 4.0 is broadly described as a suite of enabling technologies to facilitate better decision-making, predicated on the use of data [10]. This data can be sourced from simulated data and/or real production data, and is progressively available in larger volumes over time. To date, there has been limited application of Industry 4.0 (hereafter referred to as i4.0) to the composites manufacturing sector, as concluded by an industrial survey conducted by Black 2017 [15]. Published work in the literature offers insight into potential strategies and benefits of 


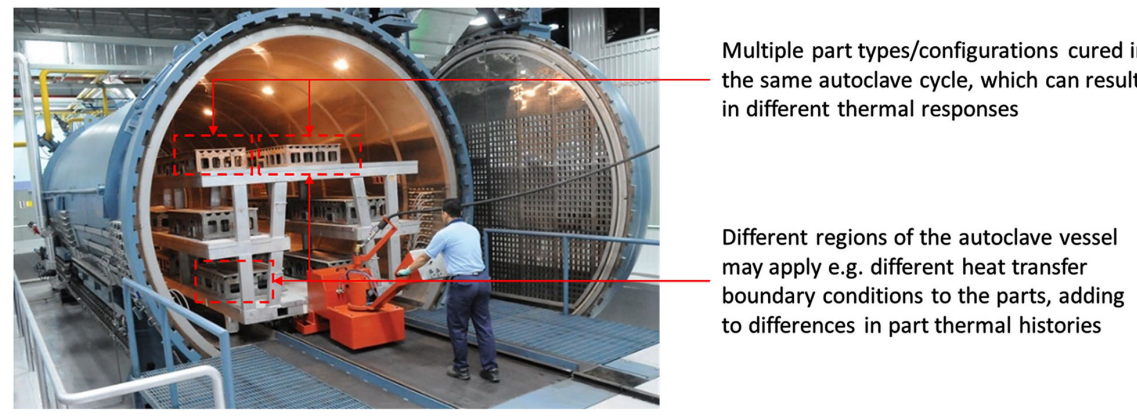

Figure 1. An autoclave used to cure structural carbon-fibre reinforced polymer composites parts. Here, the parts are arranged in a "bus-stop" cure run, where a combination of many tools and parts are loaded into the autoclave and subjected to the same cure cycle [14].

applying i4.0 principles to composites manufacturing, such as the tracking of materials and consumables throughout the manufacturing cycle [16], but still lacks a comprehensive framework for implementation on the scales of automation and broad decision-making that is seemingly promised. Such systems include different components that act in concert to collect and store data, maintain data integrity, provide predictive capabilities, with validation or qualification protocols for deployment [17].

A current lack of broader i4.0 systems in research or application settings is in part attributed to the need for further developments of the fundamental supporting technologies, including system models [18], cyber-physical systems that people can use and interact with [19], methods for model validation and qualification for in-situ use [11], among others. This convolution of challenges has led to many gaps that remain unsolved, in the development of Industry 4.0 tools and systems. Focusing on the present example of bus-stop autoclave processing, the goal for a manufacturer using i 4.0 would be to select a set of parameters in-situ that maximize the likelihood of all member parts passing the run (via qualification framework-based key process outcomes or other quality metrics). This may be necessary due to changing production priorities, accommodating new part production coming on-line, or other logistical changes. In this scenario, it is assumed that only similar parts, with marginal differences in their input configurations, would be candidates for inclusion or substitution in the bus-stop selection exercise. Such production flexibility can offer savings of time, energy and cost, via higher net part throughput rates by fully-stacking the cure vessel, increased equipment load factors that reduce the per-part rate for re-heating of the working fluid and autoclave vessel, associated labour, and other factors. This combined reduction of risk along with increased general productivity offered by i4.0 has made the technology attractive to many other industries such as health [20] and automotive [17]. However, despite the ostensible benefits, there remain many challenges in the technology's widespread implementation, especially for specific use-cases such as in advanced composites manufacturing.

The structure of this perspective paper is as follows. Section 2 provides a back- 
ground and literature review to the problem towards Composites 4.0, focusing on the fundamentals of autoclave composites manufacturing and the inherent need for qualification and explainable frameworks (Section 2.1), followed by a broader discussion on the need for model knowledgeability and explainability frameworks in machine learning (Section 2.2), and current state-of-the-art that uses machine learning models in advanced composites processing (Section 2.3). Section 3 includes concluding remarks.

\section{Towards Composites 4.0}

\subsection{Process Outcome Prediction towards Risk Reduction in Aerospace Composites Manufacturing}

Autoclave composites curing (as a sample process selected for this study) is known to be a highly complex, non-linear and multi-physics manufacturing system [1] [2], fraught with aleatoric and epistemic uncertainties that ultimately can result in risks that undermine the consistent production of high-quality aerospace parts. To increase the robustness of this process, a framework of regulations, guidelines, best-practice handbooks and other sources currently provide manufacturers with a tractable methodology for assessing and deciding on parts acceptability [9]. To this end, a key process outcome for epoxy matrix pre-impregnated advanced composites for the aerospace industry, is thermal history [9]. It is important that manufactured composite structures undergo a specific thermal history, in order to ensure that many properties of the material evolve in a controlled manner throughout the production cycle. Figure 2 provides an example thermal envelope for a carbon-epoxy prepreg, consisting of heating ramp, hold and cooling ramp segments. This processing window describes the allowable range of temperatures that a part can experience at a given time in the processing cycle, to achieve minimum performance requirements, as per the associated material and processing specification. Two key processing outcomes that can be tracked to ensure that quality metrics are maximized from a thermal processing perspective are the "peak exotherm" and "steady-state lag" tempertures. The former describes the hottest temperature experienced by the composite material throughout the manufacturing cycle, due to the combination of external thermal energy imparted by the working fluid surrounding the part, as well as the internal heat generation due to cure. Conversely, the latter is the steady-state difference in temperature between the working fluid and the composite part at the end of the ramp, when the difference is at its maximum.

A major reason for the use of thermal history-based specifications is to ensure that defects, either physical, chemical, or structural in nature, can be minimized and that material property targets are met. For example, to allow for the removal of entrapped air and outgassing from the resin for the reduction of porosity, the resin viscosity must be sufficiently low for an acceptable amount of time, which is primarily a function of temperature and degree of cure [21]. With respect to the consequences of porosity on mechanical properties, interlaminar shear 


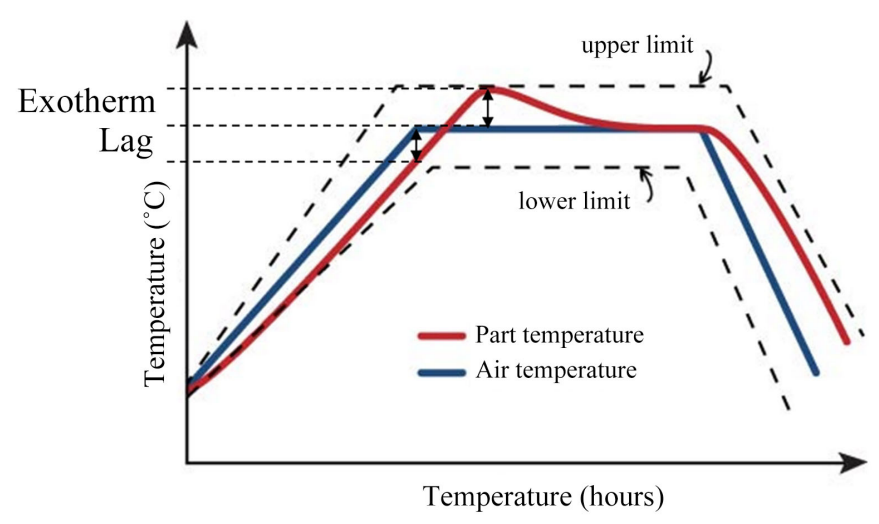

Figure 2. An example of a one-hold cure cycle, illustrating the peak exotherm and lag temperatures for the composite part, which are considered key process outcomes in determining the quality of the process in aerospace manufacturing.

strength can be reduced by approximately $7 \%$ for each $1 \%$ of void content present up to a maximum of about $4 \%$ [1], and experimental studies have shown that for a $1 \%$ increase in void content, the flexural strength, flexural modulus, and inter-laminar shear strength can decrease more than 5\% [4]. As primary aerospace structures require porosity volume fraction $\mathrm{Vf}<1 \%$, it is critical that these process bounds be adhered to, as increased porosity causes a significant reduction in mechanical properties [9].

Despite the physics-informed need to measure, identify and control process pertinent variables to avoid the generation of defects, the challenge is further compounded by realities of the manufacturing and qualification processes themselves. Namely, the need for homogeneous heating of the parts, in combination with local temperature detection, to deliver the required information for any kind of machine learning approach, is severely limited, given the intrusive nature of qualified, yet highly-local, measurement techniques (i.e. thermocouples). Chen et al. 2020 employed three years of primary structure production data combined with a meta-learning approach composed of seven machine learning-based models, thus reducing over-fitting of the meta-model, for automatic feature extraction and thermal cycle outcome prediction and recommendation, representing the volume of data required for such a task [22]. Comparatively, vision and thermal inspection systems produce data that are prime candidates for data analytics and machine learning, given the high volumes of embedded information that can effectively train, and in turn be exploited, by such models [23] [24]. For example, Manohar et al. used high-fidelity spatial scanning historical data, associated with shims used in component assembly and tolerance-based fits, used in 54 representative Boeing commercial aircraft. This data was deployed to train models that identified the locations of optimal sparse sensors (forming optimal measurement sensor ensembles) for predicting gaps to be shimmed. These optimal sensor ensembles then maximally inform the lay-up patterns used in future aircraft using sparse optimization techniques [25]. Such analogously enabling technologies in the context of autoclave cure processing 
are currently absent and largely underpin the lack of direct progress in this area.

In pursuit of minimizing defects, the metrics of peak exotherm and peak steady-state lag temperatures have been useful as key process outcomes [9]. Further, statistical structural performance estimates are provided for design activities using the composite material/processing combination, based on data collected via the building-block approach (BBA) method for material and process qualification, often in the form of A-basis and B-basis values [9].

Within the current best practice, the combination of qualification frameworks, along with the statistically-derived design allowable, forms the static, closed-loop approach to address and minimize risk in this complex system, by simultaneously constraining the materials, processes and other pertinent features. This restriction on the design space for the material, process, equipment, tooling, consumables and other elements of the factory process, works to reduce variability and uncertainty. However, this approach is typically very onerous from a time and cost perspective, while also having limited flexibility once established [26]. Such inflexibility can present challenges for shop floor decision-making contexts, such as the planning of bus-stop autoclave cures, in which multiple parts of similar features (and hence a similar expected thermal response) are loaded together into the chamber and cured in the same cycle [26]. There are many historical and ongoing efforts in this area that seek to extend and optimize the same frameworks, including developing and integrating higher-fidelity numerical models to reduce the cost of the BBA [27], reducing the conservatism and hence cost of A-basis and B-basis design allowable [28], developing consortia to share the burdens of e.g. qualifying materials [29] or design best-practices [30], among others.

The use of i4.0 seeks to provide manufacturers with opportunities for assessing a dynamic production environment, by leveraging accrued and live data, for better in-situ decisions, optimizing factory performance. However, it is recognized that this is at odds with the above addressed statically-driven framework of composites materials manufacture design and process qualification. Namely, there is a recognized need for testing, validation and qualification of production models used in this environment, to ensure confidence in using the predictive models at the heart of any i4.0 system. Many such systems also use machine-learning-based models, which are often black-box in nature and present their own challenges in building confidence. This is particularly the case when the given actual system is highly complex and direct computational models (e.g. using finite element) may be highly complex, time consuming, or low in their fidelity for use in practice.

\subsection{Need for Model Knowledgeability Frameworks When Using Machine Learning}

Ultimately, machine learning (ML) models created to describe complex systems, such as those in composites manufacturing, are intended for use as decision-aid tools by operators/designers. Forecasting of future states or designing, such sys- 
tems are very costly, time consuming, and computationally intensive, often due to finite project timelines and technical constraints within industry. ML modeling can be effectively employed as an inexpensive, rapid and powerful tool to address these problems. Once validated they can also be used to reduce the number of experiments needed for process optimization, increase safety or reliability, forecast future events under different process configurations, etc. The validation of the ML models may be achieved with the results of laboratory-scale tests or actual industrial data [31] [32], which may also include big data [33]. Further, ML models trained from real data can capture higher-order effects, or implicitly account for changes in the system unforeseen by human operators [34], such as an uncontrolled decrease in the efficiency of inductive heating elements inside of an autoclave vessel, which would otherwise be neglected from the model due to complexity, or remain unaccounted for entirely.

However, many ML models are black-box in nature; that is, black-box models are observation-based, often using experimental or observed data [35]. Unlike white-box approaches, the latter models provide low granularity, where only the data points used to train the system are considered as ground-truths along with the continuous potential predictions, let alone considering the reliability of the data used to train the model in the first place. Similarly, the domain coverage for predictions can only be reliably extended to the domain of the experimental data used to train the model in the first place. Black-box models attempt to encode the relationships between variables, by minimizing or maximizing an objective function (e.g. mean-squared error between observations and predictions) through a training process (e.g. gradient descent implemented through backpropagation). This relationship may not even be physics-based, depending on the inputs and outputs of the system, as chosen by the user. Hence, disparate model inputs may be used, which have no inherent direct physical relationship that can be quantified, as is characteristic of heuristic models. Additionally, the accuracy of such models is subject to many constraints, including the volume, sparsity and reliability of the available data. Figure 3 provides an overview of the trade-off between different model types, between the black- and white-box regimes.

Despite the advantages of using black-box ML in production environments, significant challenges remain, including their lack of interpretability. As Rudin et al. 2019 claim, any model that automatically "learns" from a dataset and is not manually constructed, has the potential to be intractable at any point in its domain, by virtue of the model builder implicitly not being hands-on in that process [36]. This perspective may be particularly relevant for use cases with limited data available, due to there being less embedded statistical information, further relying on the performance of increasingly complex and high-fidelity models to compensate [37]. Recent developments seeking to retain the advantages of black-box ML, while increasing model confidence, include the strategic drafting of federal policy towards fostering scientific machine learning (SciML) in which scientific principles can be more tractably encoded into such models 


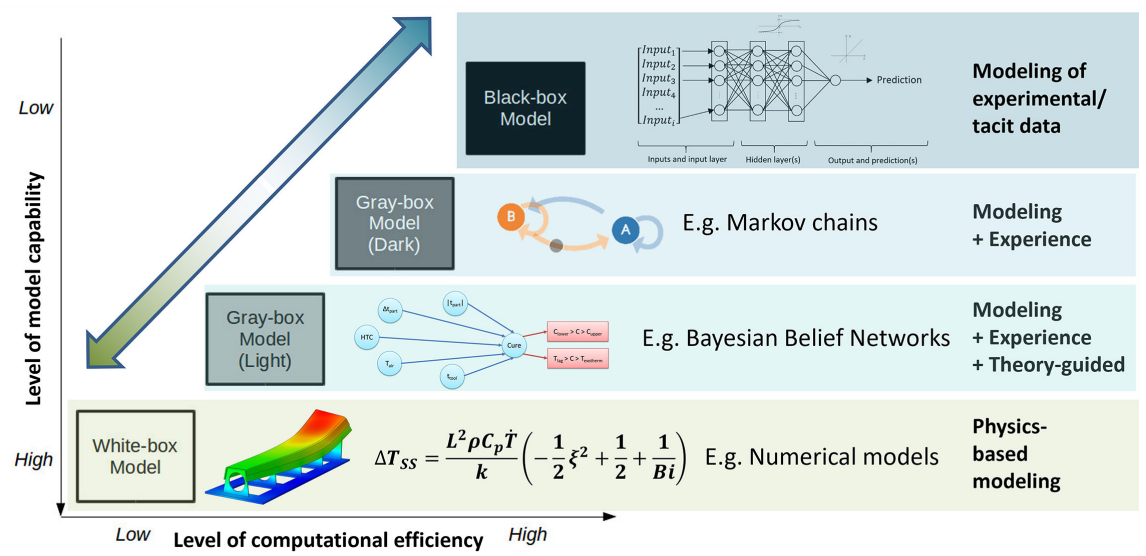

Figure 3. An illustration of the continuum between white-box and black-box modeling of thermal management in composites manufacturing. White-box models are rooted in physics; in this case the prediction of the steady-state temperature lag between the heated working fluid and a monolithic and isotropic material can be estimated as a function of the material geometry, properties and applicable descriptive dimensionless numbers (i.e. here the Biot number). However, black-box models, such as Artificial Neural Networks can also be trained to provide predictive capabilities for the same system.

[38]. Iten et al. 2020 demonstrated this approach by leveraging observational data to encode a deep ANN, parameterize the data via a latent representation layer, followed by decoder ANN, called SciNet [39]. This approach has been employed in process modeling for manufacturability assessments in the preliminary design of autoclave-cured composite laminates, which provided greater prediction accuracy of the thermal processing outcomes compared to traditional methods [40] [41]. Giving model designers the ability to direct models to learn from datasets predicated on physics-based relationships (e.g. non-dimensional numbers) allows for greater tractability and interpretability in how the model constructs its representations of the complex curing scenario.

Beyond capturing physics-based phenomena, mathematical processes computed by an ANN developed by Lample et al. 2020, are able to symbolically compute function integrals and solve complex differential equations [42]. AI interpretability tools have also emerged as methods for application-based investigation of black-box ML, leveraging approaches such as constructing local models, or Shapley values (solution concept in cooperative game theory) to provide users with expected values or losses attributed to changes within the feature space [43] [44] [45] [46]. Further, the integration of heuristic methods with classical machine learning algorithms has offered higher model performance in various applications. For example, Geirhos et al. 2019 demonstrated that in computer vision classification-based tasks, ImageNet-trained CNNs are strongly biased towards using information from textures, rather than edges and shapes. This is a fundamentally different strategy for classification, when compared to human behavioral evidence, whereby incorporating strategies more in line with the latter ultimately improved model performances [47]. This provides further impetus for deeper user involvement in the construction and evaluation of 
black-box models.

\subsection{Current State-of-the-Art for Machine Learning in Composites}

With the emergence of increasingly robust and versatile machine learning models and open-source tools for their implementation, there has been growth in the volume of publications in the open literature that investigate the use of ML models in composites manufacturing and design. Here, a selection of the research has been reviewed and the results discussed, in the context of this study. It should be noted that research into the application of ML specifically in composites manufacturing is sparse. As such, select systems have been successfully modeled using this strategy and few critical comparisons between studies can be made. Studies include modeling the production of carbon fibre [48] [49], optimization of the automated fibre placement process [50], modeling of the dynamic cure process [51], as well as the assessment of defects for quality control, namely fibre orientation [52] and delamination [53] [54].

Khayyam et al. studied the use of various predictive models that were applied to the carbon fibre production process, specifically the stabilization step of the process. Gaussian-Newton, Levenberg-Marquardt Neural Network (LM-ANN) and Taylor series methods were used to model and predict the Carbon Fibre heat of reaction and modulus, as process outcomes from the stabilization step [48]. This study was extended to include the polyacrylonitrile (PAN) precursor fabrication process [49] [50], with Support Vector Machine (SVM)-based models also studied in [51]. The latter studies demonstrated that the machine learning model choices can be well relevant to the larger distributed supply chains. Further, the inclusion of multi-objective design optimization techniques under considerations of uncertainty extended these models to broader industrial applications [52] [53].

Brüning et al. 2017 proposed a method towards the optimization of the automated fiber placement processes, using ML predictive models. The process variables considered include temperature, compaction pressure and lay-up head velocity. Multiple linear regression models were used for any predictive work, which was supported by the development of an XML database for in-situ process monitoring [54].

Modeling of the autoclave cure process using black-box approaches has received some attention. Namely, Carlone et al. 2018 showed that dynamic predictions of the process can be made using Recurrent Neural Networks (RNNs), using the Long-Short Term Memory (LSTM) architecture [55]. This model was trained on pseudo-experimental (virtual data) and was able to capture the time-dependent nature of this complex system. This was performed in the context of the composite parts containing complex 3D geometries and having different fibre-volume fractions, where the thermal history of the composite throughout the cure cycle, is the model output. In their study, the Levenberg-Marquardt (LM), Bayesian Regularization (BR), Resilient Backpropagation (RP), and Scaled 
Conjugate Gradient (SCG) training functions were compared, where the ANN model based on BR provided the best performance.

There has been greater interest in the use of ML for in-situ defect estimation for quality control purposes in composites manufacturing, which is reflected by the greater volume of papers in this area. For example, Schmitt et al. [56] studied the feasible production of fiber-reinforced composites through inline inspection with machine vision. This work used a Fourier transform approach to classify local regions of an image, to determine the fibre/textile type, in order to catch lay-up errors, where technicians or other soft factors may have led to the defect. The study was set-up as a preface to the future application of ML models, as no ML algorithms were performed. Later, the work in this area was further developed by Hernán et al. [57], by detecting delamination-based defects using infrared thermography. Using the thermography images of composites as model inputs, dynamic PCA and K-NN models were used in an unsupervised learning regime to determine structure in the data as a dimensionally-reduced input into further ML models. Specifically, the Radial Basis Function was used as the kernel function in a trained SVM model, to detect and quantify the defect depth in the composite parts. Further, Fernandes et al. [58] recently investigated defect characterization in infrared thermography-based non-destructive testing (NDT), supported by ML for the prediction of fibre orientation. Pulsed thermal ellipsometry was the NDT method employed to capture images of internal features of the composite, which was combined with ANN models to estimate the fiber orientation on the surface of inspected parts. The authors commented on the good accuracy and speed of their approach, which in the context of the current industrial landscape, is a core requirement for the successful implementation of Industry 4.0 .

\subsection{Future Perspectives}

Research in the wider literature has demonstrated successful applications of machine learning as a means for the modeling of composites production processes, for both prognostic design or in-situ defect characterization purposes. While this provides basic confidence in the capabilities of this approach, there remains concerns with model tractability and explainability for use in industrial applications, resting in a user's ability to independently demonstrate how and why black-box ML models generate their outputs. In this context, many gaps and opportunities remain for further research in building ML-based models for use in composite manufacturing environments, which meet the human-interpretable needs for tractable risk reduction. Recent advances in pre-processing datasets, such as physics-informed ML [40], has provided increased interpretability for model designers by directly influencing the learning process in a common physics-based framework, towards using models for interpolation-based predictions. Further, with the recent emergence of AI explainability tools such as Local Interpretable Model-Agnostic Explanations (LIME)-based toolboxes, or the Shapley val- 
ue-based approach used by Fiddler Labs [43], there is an opportunity for further research in this field to address the above concerns, in both the construction and evaluation of models, and associated datasets for advanced manufacturing. These advanced, combined with government-led strategic efforts to actively foster applied research in this area, has supported the framework and impetus for integrating Industry 4.0 principles in advanced composites manufacturing.

\section{Conclusion}

Aerospace autoclave composites manufacturing is a highly complex process, which is guided by a framework of risk-based decision-making (which is done often off-line using process numerical models) for qualification and proper use of materials and tools, and ultimately arriving at defect-free parts. This framework is meant to introduce some measure of inflexibility (tight tolerances) into the underlying industrial process parameters (e.g. to stay within allowable peak exotherm and lag temperatures), in exchange for providing higher confidence in delivering high-quality (defect-free) parts. Industry 4.0 is a broadly-defined suite of technologies and tools that are used to the same end of reducing risk and increasing the likelihood of success in production, albeit in a dynamic and in-situ role, to be used to inform decision-makers on the manufacturing shop floor. This may be viewed at odds with the traditional qualification framework, especially given that models at the core of i4.0 are data-driven and updatable in real-time. Current trends and future opportunities in the wider research have illustrated both the potential and the need for introducing new methods that ultimately reduce the risk of advanced manufacturing activities under the new Industry 4.0 paradigm. Within this trend, emphasizing the interpretability of the prediction models, and pre-assessment of "knowledgeability" of given data prior to training are deemed central to successful implementations of Industry 4.0.

\section{Conflicts of Interest}

The authors declare no conflicts of interest regarding the publication of this paper.

\section{References}

[1] Strong, A.B. (2008) Fundamentals of Composites Manufacturing: Materials, Methods and Applications. Society of Manufacturing Engineers, Southfield.

[2] Mazumdar, S.K. (2002) Composites Manufacturing: Materials, Product, and Process Engineering. CRC Press, Boca Raton. https://doi.org/10.1201/9781420041989

[3] Mangalgiri, P. (1999) Composite Materials for Aerospace Applications. Bulletin of Materials Science, 22, 657-664. https://doi.org/10.1007/BF02749982

[4] Mesogitis, T.S., Skordos, A.A. and Long, A.C. (2014) Uncertainty in the Manufacturing of Fibrous Thermosetting Composites: A Review. Composites Part A: Applied Science and Manufacturing, 57, 67-75. https://doi.org/10.1016/j.compositesa.2013.11.004

[5] Fernlund, G. (2010) Risk Reduction in Composites Processing Using Prototype Da- 
ta, Process Simulation, and Bayesian Statistics. Composites Part A: Applied Science and Manufacturing, 41, 295-303. https://doi.org/10.1016/j.compositesa.2009.10.021

[6] Potter, K. (2009) Understanding the Origins of Defects and Variability in Composites Manufacture. Proceedings of the International Conference on Composite Materials (ICCM)-17, Edinburgh, 27-31 July 2009.

[7] Convergent (2018) COMPRO Simulation Software. https://www.convergent.ca/products/compro-simulation-software

[8] Esi-Group (2018) PAM-RTM -Confidently Build Lightweight Composite Products Free From Defects with Easy-to-Use Composite Simulation Software. https://www.esi-group.com/software-solutions/virtual-manufacturing/composites/p am-composites/pam-rtm-composites-molding-simulation-software

[9] Revision, G. (2012) Composite Materials Handbook, Volume 3: Polymer Matrix Composites Materials Usage, Design and Analysis (CMH-17). SAE International, Wichita.

[10] Dynamiccio (2018) Neither C-Suite Nor Millennial Workforce Feel Ready and Confident for Industry 4.0.

https://www.dynamiccio.com/neither-c-suite-nor-millennial-workforce-feel-readyand-confident-for-industry-4-0/

[11] Xu, L., Xu, E. and Li, L. (2018) Industry 4.0: State of the Art and Future Trends. International Journal of Production Research, 8, 2941-2962.

https://doi.org/10.1080/00207543.2018.1444806

[12] McKinsey \& Company (2013) Game Changers: Five Opportunities for US Growth and Renewal.

https://www.mckinsey.com/featured-insights/americas/us-game-changers

[13] McAfee, A., Brynjolfsson, E., Davenport, T.H., Patil, D. and Barton, D. (2012) Big Data. The Management Revolution. Harvard Business Review, 90, 60-66.

[14] Polek, G. (2014) For Boeing and Airbus, Partnerships Proliferate in Asia. https://www.ainonline.com/aviation-news/aerospace/2014-02-10/boeing-and-airbus -partnerships-proliferate-asia

[15] Black, S. (2017) Composites and Industry 4.0: Where Are We? https://www.compositesworld.com/articles/composites-and-industry-40-where-arewe

[16] Al-Lami, A. and Hilmer, P. (2016) Novel Applications in Assessing Manufacturing and Assembly of Complex Composite Structures: A Pace towards Industry 4.0 in Composite Manufacturing. Proceedings of the 11 th AIRTEC Congress, Munich, 27 October 2016.

[17] Ayafrudin, M., Alfian, G., Fitriyani, N.L. and Rhee, J. (2018) Performance Analysis of IoT-Based Sensor, Big Data Processing, and Machine Learning Model for Real-Time Monitoring System in Automotive Manufacturing. Sensors, 18, 2946-2972. https://doi.org/10.3390/s18092946

[18] Campos, J. (2010) Guest Editorial Special Section on Formal Methods in Manufacturing. IEEE Transactions on Industrial Informatics, 2, 125-126. https://doi.org/10.1109/TII.2010.2042529

[19] National Science Foundation (2019) Cyber-Physical Systems (CPS). https://www.nsf.gov/funding/pgm_summ.jsp?pims_id=503286

[20] Roski, J., Bo-Linn, G.W. and Andrews, T. (2014) Creating Value in health Care through Big Data: Opportunities and Policy Implications. Health Affairs, 33, 1115-1122. https://doi.org/10.1377/hlthaff.2014.0147 
[21] Johnston, A.A. (1997) An Integrated Model of the Development of Process-Induced Deformation in Autoclave Processing of Composite Structures. Ph.D. Dissertation, University of British Columbia, Vancouver, Kelowna.

[22] Chen, Y., Wang, L. and Chu, P. (2020) A Recipe Parameter Recommendation System for an Autoclave Process and an Empirical Study. Procedia Manufacturing, 51, 1046-1053. https://doi.org/10.1016/j.promfg.2020.10.147

[23] Brunton, S.L. and Kutz, J.N. (2019) Data-Driven Aerospace Engineering: Reframing the Industry with Machine Learning. Cambridge University Press, Cambridge, 1-35. https://doi.org/10.1017/9781108380690

[24] Sacco, C., Radwan, A., Beatty, T., Harik, R. (2020) Machine Learning Based AFP Inspection: A Tool for Characterization and Integration. Proceedings of the SAMPE 2019 Conference, Charlotte, 20-23 May 2019. https://doi.org/10.33599/nasampe/s.19.1594

[25] Manohar, K., Hogan, T., Buttrick, J., Banerjee, A., Kutz, J.N. and Brunton, S. (2018) Predicting Shim Gaps in Aircraft Assembly with Machine Learning and Sparse Sensing. Journal of Manufacturing Systems, 48, 87-95. https://doi.org/10.1016/j.jmsy.2018.01.011

[26] Hueber, C., Fischer, G., Schwingshandl, N. and Schledjewski, R. (2009) Production Planning Optimization for Composite Aerospace Manufacturing. International Journal of Production Research, 57, 5857-5873. https://doi.org/10.1080/00207543.2018.1554918

[27] Abdi, F., Surdenas, J., Munir, N., Housner, J. and Keshavanarayana, R. (2009) Computational Approach toward Advanced Composite Material Qualification and Structural Certification. In: Farahmand, B., Ed., Virtual Testing and Predictive Modeling, Springer, Boston, 137-185. https://doi.org/10.1007/978-0-387-95924-5_6

[28] Abdi, F., Clarkson, E., Godines, C. and DorMohammadi, S. (2016) A-B Basis Allowable Test Reduction Approach and Composite Generic Basis Strength Values. Proceedings of the 18th AIAA Non-Deterministic Approaches Conference, San Diego, 4-8 January 2016. https://doi.org/10.2514/6.2016-0951

[29] Lee, H.S., Rhee, S.Y., Yoon, J.K., Yoo, J.T. and Min, K.J. (2015) Establishment of Aerospace Composite Materials Data Center for Qualification. Composites Research, 6, 402-407. https://doi.org/10.7234/composres.2015.28.6.402

[30] Berenberg, B. (2003) AGATE Methodology Proves Its Worth. https://www.compositesworld.com/articles/agate-methodology-proves-its-worth

[31] Rodrigues, A.E. and Minceva, M. (2005) Modelling and Simulation in Chemical Engineering: Tools for Process Innovation. Computers \& Chemical Engineering, 29, 1167-1183. https://doi.org/10.1016/j.compchemeng.2005.02.029

[32] Dobre, T.G. and Sanchez-Marcano, J.G. (2007) Chemical Engineering: Modelling, Simulation and Similitude. Wiley-VCH Verlag GmbH \& Co. KGaA, Weinheim. https://doi.org/10.1002/9783527611096

[33] Qiu, J., Wu, Q., Ding, G., Xu, Y. and Feng, S. (2016) A Survey of Machine Learning for Big Data Processing. EURASIP Journal on Advances in Signal Processing, 2016, Article No. 85. https://doi.org/10.1186/s13634-016-0382-7

[34] Wuest, T., Weimer, D., Irgens, C. and Thoben, K. (2016) Machine Learning in Manufacturing: Advantages, Challenges, and Applications. Production \& Manufacturing Research, 4, 23-45. https://doi.org/10.1080/21693277.2016.1192517

[35] Khan, M.E. and Khan, F. (2012) A Comparative Study of White Box, Black Box and Grey Box Testing Techniques. International Journal of Advanced Computer Science 
and Applications, 3, 12-25. https://dx.doi.org/10.14569/IJACSA.2012.030603

[36] Rudin, C. (2019) Stop Explaining Black Box Machine Learning Models for High Stakes Decisions and Use Interpretable Models Instead. Nature Machine Intelligence, 1, 206-215. https://doi.org/10.1038/s42256-019-0048-x

[37] Khayyam, H., Jamali, A., Bab-Hashidar, A., Esch, T., Ramakrishna, S., Jalili, M., et al. (2020) A Novel Hybrid Machine Learning Algorithm for Limited and Big Data Modelling with Application in Industry 4.0. IEEE Access, 8, 111381-111393. https://doi.org/10.1109/ACCESS.2020.2999898

[38] Baker, N., Alexander, F., Bremer, T., Hagberg, A., Kevrekidis, Y., Habib, N., et al. (2019) Workshop Report on Basic Research Needs for Scientific Machine Learning: Core Technologies for Artificial Intelligence. Office of Scientific and Technical Information, Oak Ridge.

[39] Iten, R., Metger, T., Wilming, W., del Rio, L. and Renner, R. (2020) Discovering Physical Concepts with Neural Networks. Physical Review Letters, 124, 105-129. https://doi.org/10.1103/PhysRevLett.124.010508

[40] Zobeiry, N. and Humfeld, K.D. (2020) A Physics-Informed Machine Learning Approach for Solving Heat Transfer Equation in Advanced Manufacturing and Engineering Applications. Engineering Applications of Artificial Intelligence, 101, Article ID: 104232. https://doi.org/10.1016/j.engappai.2021.104232

[41] Zobeiry, N., Van Ee, D.A. and Poursartip, A. (2019) Theory-Guided Machine Learning Composites Processing Modelling for Manufacturability Assessment in Preliminary Design. Proceedings of the NAFEMS 17 th World Congress, Quebec City, 17-20 June 2019.

[42] Lample, G. and Charton, F. (2020) Deep Learning for Symbolic Mathematics. Proceedings of International Conference on Learning Representations, Virtual conference, Addis Ababa, 26 April-1 May 2020.

[43] Mojsilovic, A. (2019) Introducing AI Explainability 360. https://www.ibm.com/blogs/research/2019/08/ai-explainability-360/

[44] Google (2019) AI Explainability Whitepaper.

[45] Riberio, M., Singh, S. and Guestrin, C. (2016) "Why Should I Trust You?": Explaining the Predictions of Any Classifier. Proceedings of the 22nd ACM SIGKDD International Conference on Knowledge Discovery and Data Mining, San Francisco, 13-17 August 2016, 1135-1144. https://doi.org/10.1145/2939672.2939778

[46] Merrick, L. and Taly, A. (2020) The Explanation Game: Explaining Machine Learning Models Using Shapley Values. Proceedings of the International Cross-Domain Conference for Machine Learning and Knowledge Extraction, Dublin, 25-28 August 2020, 17-38. https://doi.org/10.1007/978-3-030-57321-8_2

[47] Geirhos, R., Rubisch, P., Michaelis, C., Bethge, M., Wichmann, F. and Brendel, W. (2019) ImageNet-Trained CNNs Are Biased towards Texture; Increasing Shape Bias Improves Accuracy and Robustness. Proceedings of the International Conference on Learning Representations, New Orleans, LA, 6-9 May 2019, 22.

[48] Khayyam, H., Naebe, M., Zabihi, O., Zamani, R., Atkiss, S. and Fox, B. (2015) Dynamic Prediction Models and Optimization of (PAN) Stabilization Processes for Production of Carbon Fiber. IEEE Transactions on Industrial Informatics, 11, 887-896. https://doi.org/10.1109/TII.2015.2434329

[49] Khayyam, H., Jazar, R., Nunna, S., Golkarnarenji, G., Badii, K., Fakhrhoseini, S., et al. (2020) PAN Precursor Fabrication, Applications and Thermal Stabilization Process in Carbon Fiber Production: Experimental and Mathematical Modelling. Progress 
in Materials Science, 107, 100575-100593.

https://doi.org/10.1016/j.pmatsci.2019.100575

[50] Golkarnarenji, G., Naebe, M., Church, J.S., Badii, K., Bab-Hadiashar, A. and Atkiss, S. (2017) Development of a Predictive Model for Study of Skin-Core Phenomenon in Stabilization Process of PAN Precursor. Journal of Industrial and Engineering Chemistry, 49, 46-60. https://doi.org/10.1016/j.jiec.2016.12.027

[51] Golkarnarenji, G., Naebe, M., Badii, K., Milani, A.S., Jazar, R.N. and Khayyam, H. (2018) Support Vector Regression Modelling and Optimization of Energy Consumption in Carbon Fiber Production Line. Computers \& Chemical Engineering, 109, 276-288. https://doi.org/10.1016/j.compchemeng.2017.11.020

[52] Ramezankhani, M., Crawford, B., Khayyam, H., Naebe, M., Seethaler, R. and Milani, A.S. (2019) A Multi-Objective Gaussian Process Approach for Optimization and Prediction of Carbonization Process in Carbon Fiber Production under Uncertainty. Advanced Composites and Hybrid Materials, 2, 444-455.

https://doi.org/10.1007/s42114-019-00107-6

[53] Golkarnarenji, G., Naebe, M., Badii, K., Milani, A.S, Jamali, A., Bab-Hadiashar, A. and Khayyam, H. (2019) Multi-Objective Optimization of Manufacturing Process in Carbon Fiber Industry Using Artificial Intelligence Techniques. IEEE Access, 7, 67576-67588. https://doi.org/10.1109/ACCESS.2019.2914697

[54] Brüning, J., Denkena, B., Dittrich, M.A. and Hocke, T. (2017) Machine Learning Approach for Optimization of Automated Fiber Placement Processes. Procedia CIRP, 66, 74-78. https://doi.org/10.1016/j.procir.2017.03.295

[55] Carlone, P., Aleksendrić, D., Rubino, F. and Ćirović, V. (2018) Artificial Neural Networks in Advanced Thermoset Matrix Composite Manufacturing. Proceedings of the 3 rd International Conference on the Industry 4.0 Model for Advanced Manufacturing, Belgrade, 5-7 June 2018, 78-88.

https://doi.org/10.1007/978-3-319-89563-5_5

[56] Schmitt, R., Orth, A. and Tilo, P. (2006) Feasible Production of Fiber-Reinforced Composites through Inline Inspection with Machine Vision. Proceedings of the IMEKO XVIII World Congress and IV Brazilian Congress of Metrology, Rio de Janeiro, 17-22 September 2006.

[57] Hernán, D., Loaiza., B.H., Caicedo, E., Ibarra-Castanedo, C., Bendada, A.H. and Maldague, X. (2009) Defect Characterization in Infrared Non-Destructive Testing with Learning Machines. NDT \& E International, 42, 630-643.

https://doi.org/10.1016/j.ndteint.2009.05.004

[58] Fernandes, H., Zhang, H., Figueiredo, A., Malheiros, F., Ignacio, L.H., Sfarra, S., et al, (2018) Machine Learning and Infrared Thermography for Fiber Orientation Assessment on Randomly-Oriented Strands Parts. Sensors, 18, 288-298.

https://doi.org/10.3390/s18010288 